\title{
Revisão
}

\section{Tendencias de las publicaciones especializadas en el campo de la educomunicación y alfabetización mediática en Latinoamérica}

\author{
Research trends in specialist publications from the field of \\ educommunication and media literacy in Latin America (abstract: p. 16)
}

Tendências das publicações especializadas no campo da educomunicação e alfabetização midiática na América Latina (resumen: p. 17)

\author{
Gabriel Lotero-Echeverri(a) \\ <gabrielj.lotero@upb.edu.co> \\ Luis M. Romero-Rodríguez ${ }^{(\mathbf{b})}$ \\ <luis@romero-rodriguez.com> iD
}

Amor Pérez-Rodríguez ${ }^{(\mathrm{c})}$

<amor@uhu.es> iD

\author{
(a) Facultad de Comunicación Social, \\ Periodismo de la Universidad \\ Pontificia Bolivariana de Medellín \\ (Colombia). Circular 1 N $70-01$. \\ Medellín, Colombia. \\ (b) ESAI Business School, Universidad \\ Espíritu Santo (Ecuador). Guayaquil, \\ Ecuador. \\ (c) Universidad de Huelva. Huelva, \\ España.
}

Se analizan tendencias recientes de investigación en comunicación publicadas en revistas de comunicación latinoamericanas más visibles en bases de datos de referencia y citación. La revisión se centra en los artículos de investigación publicados entre 2013 y 2017 en las nueve revistas latinoamericanas de comunicación incluidas en Scopus. La metodología se basa en la consulta de bases de datos, su sistematización a través de gestores bibliográficos y el análisis de contenido cualitativo de los textos recopilados, apoyado con el software de QDA NVIV011. Se recopilan 167 artículos que abordan temáticas de educomunicación, alfabetización mediática y narrativas transmedia. Predomina la autoría individual y la afiliación a un solo país e institución, entre los cuales destacan Brasil y España. En relación con el contenido, predomina un enfoque temático sobre los medios y los entornos digitales y un abordaje cualitativo de las investigaciones.

Palabras clave: Comunicación. Educación. Educomunicación. Alfabetización mediática. Revista científica. 


\section{Introducción}

El presente texto tiene como propósito revisar las revistas del campo de la comunicación, editadas en Latinoamérica, que son más visibles en bases de datos internacionales de referencia y citación, como la base de datos Scopus y Web of Science. De igual forma, se revisa la situación de las revistas seleccionadas en otras bases de datos, como Scielo y Redalyc y su visibilidad en el Google Scholar Metrics, a través del índice H-5.

El propósito es identificar tendencias en el abordaje de temáticas propias de la dinámica relación entre la comunicación y la educación en la contemporaneidad, la cual se ve atravesada por la dimensión digital, que representa un campo de oportunidades y desafíos. No se conocen revisiones que profundicen en este campo y que cubran una muestra tan amplia de artículos y revistas latinoamericanas, que cuenten con indicios de calidad, como su inclusión en bases de datos de referencia internacional.

La revisión se centra en los artículos de investigación publicados en las nueve revistas latinoamericanas incluidas en Scopus ${ }^{(\mathrm{d})}$, siete de las cuales también se encuentran en Scielo y se analizan los años entre 2013 y 2017.

\section{La investigación en comunicación en Latinoamérica}

El desarrollo de los estudios de comunicación ha sido acelerado, debido al interés creciente en los medios de comunicación masiva (mass media), especialmente de la prensa, la radio y la televisión. La investigación en comunicación se ha venido desarrollando como un campo de conocimiento en construcción, complejo y transdisciplinar ${ }^{1}$.

En Latinoamérica existe un campo académico de la comunicación muy importante, que incluye la formación de nuevos profesionales en grados universitarios y estudios de postgrado en gran diversidad de áreas de la comunicación. En la región latinoamericana existe una fuerte tradición en estudios universitarios en el campo de la comunicación social y el periodismo. De acuerdo con datos de la Federación Latinoamericana de Facultades de Comunicación Social ${ }^{(e)}$, existen 267 facultades y programas universitarios, del área de comunicación, están adscritos a la Federación. De estos, 253 son de Latinoamérica, diez de España, tres de Estados Unidos y uno de Canadá. En la región, el campo investigativo de la comunicación se desarrolla en gran medida en las facultades de comunicación, como un esfuerzo de los profesores y como contribución en la formación de nuevos investigadores.

González-Samé, Romero-Rodríguez y Aguaded ${ }^{2}$ afirman que no es posible hablar de una Escuela latinoamericana de Comunicación, porque en la región se han desarrollado estudios de manera dispersa, carentes de una identidad epistemológica y de uniformidad metodológica: "las investigaciones del campo se han tratado históricamente como una agrupación de estudios dispersos que se han hecho sobre cultura y comunicación en Latinoamérica” (p. 429). Afirman que la investigación en comunicación latinoamericana refleja una dependencia de tres importantes tendencias internacionales: la investigación norteamericana, predominantemente funcionalista; la teoría crítica de la escuela de Frankfurt y la escuela de los estudios culturales con importantes desarrollos en el mundo anglosajón. (d) No se incluye la revista Chasqui, porque se encuentra clasificada en otra categoría: en "artes y humanidades". Esta revista tampoco se encuentra incluida en la base de datos de Scielo.

(e) Federación Latinoamericana de Facultades de Comunicación Social. Miembros plenos - http:// felafacs.org/miembros/plenos/ y asociados - http://felafacs. org/miembros/asociados/ 
Sin embargo, a partir de los años ochenta del siglo pasado, de manera destacada desde la obra de Jesús Martín-Barbero ${ }^{3}$, es posible identificar un giro en los objetos de estudio predominantes en Latinoamérica. Martín-Barbero plantea la necesidad de trascender el énfasis de las investigaciones sobre medios masivos y tecnologías importadas, desde un enfoque predominantemente funcionalista, de manera que sea posible comprender los procesos de comunicación como mediaciones, desde la escuela de los estudios culturales, que permita visibilizar las culturas populares y los procesos de hibridación de identidades, lo cual ha marcado una tendencia importante en la investigación en comunicación en la región, tal y como lo explica Martin-Barbero en un texto reciente ${ }^{4}$ al intentar revisitar su obra para preguntarse desde dónde pensar hoy la comunicación.

Como parte de este giro cultural y a partir del desarrollo del campo universitario de la comunicación, también se destaca, como una tendencia importante en la investigación latinoamericana, la perspectiva de los estudios de la comunicación para el desarrollo y el cambio social ${ }^{5}$, desde un enfoque crítico y emancipador, consistente con las luchas sociales, los medios comunitarios y los proyectos de educación popular, orientados hacia el empoderamiento de las comunidades empobrecidas y marginadas.

Desde finales del siglo XX, el giro hacia lo cultural, sin embargo, se ve nuevamente contrastado por la creciente influencia de la popularización de las tecnologías de la información y la comunicación, por lo cual se plantea un giro hacia la comunicación digital como un objeto de estudio predominante en las investigaciones en comunicación en la región latinoamericana, como una tendencia que responde al interés por los nuevos medios digitales y las transformaciones que plantea a los modelos de comunicación, a las interacciones con y entre usuarios hiperconectados, en un proceso de convergencia tecnológica que potencia la cultura de la participación ${ }^{6}$.

\section{Comunicación y educación}

La consolidación de una sociedad de la información se basa en el acceso a cuatro servicios de conectividad: la telefonía fija, la telefonía móvil, la banda ancha fija y banda ancha móvil. Según el Informe "Sociedad de la información", en 2016 se avanzó en la conectividad mundial a través de estos servicios, que evidencia un mejoramiento en el acceso a Internet en los hogares y a nivel personal, excepto en telefonía fija, que presenta una tendencia a la baja.

Merece la pena resaltar la existencia de una importante tendencia a la sobresaturación y a usos en los que predomina un entretenimiento fácil y liviano infoxicación-. Incluso en personas de mayor nivel educativo y que se están formando como profesionales expertos en comunicación existe el riesgo de ocurrencia de estos fenómenos por el exceso de consumo de información superflua e innecesaria ${ }^{8}$.

Existe una creencia muy extendida en un fenómeno llamado «nativos digitales», que afirma, sin mayores fundamentos, que quienes nacieron en la era de Internet cuentan con las capacidades y competencias que el entorno digital exige, de manera automática.

El acceso es importante y constituye un logro de las políticas públicas que se han enfocado en facilitar que la población esté conectada, sin embargo, no es suficiente, porque también se debe contar con competencias mediáticas y digitales suficientes para 
fortalecer la apropiación social de las TIC al servicio de los procesos de educación y desarrollo humano.

Desde esta perspectiva, la Unesco ${ }^{9}$ habla de educación en materia de comunicación (media education), aunque esta línea de trabajo se ha nombrado de diversas formas: Educación para los medios, Educomunicación, Educación para la Comunicación, Educación por la Comunicación, Educación para la recepción (crítica, activa), Pedagogía de la Imagen, pedagogía para los medios, entre otras. Más allá de la educación en medios, desde un enfoque instrumental, en Latinoamérica ha tenido gran desarrollo un escenario de convergencia, en el cual se encuentra la comunicación y la educación, como un campo transdisciplinar, que Oliveira-Soares ${ }^{10}$ llama Educomunicación, en el cual los usuarios van más allá del derecho a la información y asumen un rol más activo y crítico, en procesos de relación entre medios y usuarios, más horizontales y democráticos.

Marta-Lazo ${ }^{11}$ afirma que el principal objetivo de esta propuesta es potenciar la capacidad de análisis y crítica del estudiante ante los medios de comunicación masiva. Ferrés y Piscitelli ${ }^{12}$ plantean que, considerando las características complejas de la competencia mediática, es pertinente que las propuestas educativas articulen formación en las dimensiones: del lenguaje, de la tecnología, estética, valores, procesos de producción, difusión e interacción.

\section{Procedimientos metodológicos}

Se realiza un estudio de tipo mixto de las revistas científicas de comunicación editadas en Latinoamérica, a través de la revisión de las bases de datos Scimago Journal \& Country Rank de Scopus y Journal Citation Reports de Web of Science, así como los repositorios digitales Scielo y Redalyc y la plataforma Google Scholar Metrics. Se seleccionan nueve revistas por su inclusión en la base de datos de citación Scopus y en los repositorios digitales mencionados y la valoración de su índice H-5 en Google Scholar (ver tabla 1, en el apartado de resultados).

En un primer momento, se realiza una selección de las revistas latinoamericanas de comunicación, según su inclusión en la categoría "comunicación" de las bases de datos de referencia y citación Scopus e ISI Web of Science (Wos). Se encuentran nueve revistas en Scopus y ninguna en Wos. Las nueve revistas seleccionadas son:

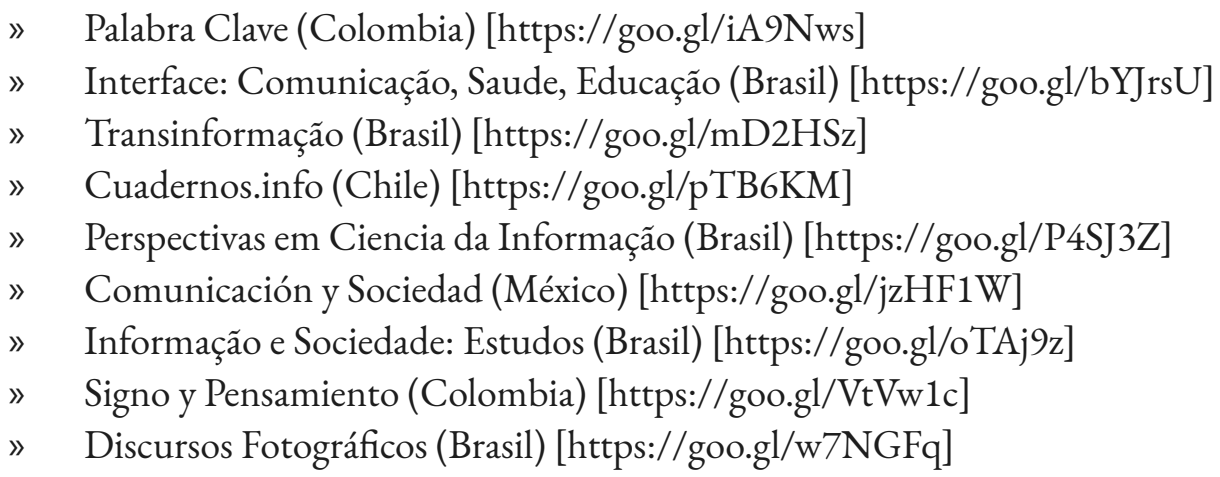

En un segundo momento, a través de la base de datos de Scopus ${ }^{(f)}$, se recuperan los artículos de investigación publicados en las nueve revistas latinoamericanas

(f) Elsevier. About Scopus https://www.elsevier.com/ solutions/scopus 
seleccionadas, durante los últimos cinco años, en el período comprendido entre 2013 y 2017; esta delimitación se justifica debido al elevado número de artículos y también porque permite identificar tendencias más recientes en cuanto a los objetos de estudio adordados. Los artículos son recopilados y sistematizados en el gestor bibliográfico Mendeley.

Para la selección de los artículos, se utilizan filtros y algoritmos booleanos para limitar los resultados a los que estén clasificados como "artículos de investigación” y que estén archivados con las palabras clave, indicadas a través de casillas de selección múltiple:

- Access

- Cultural

- $\quad$ Audiences

- Citizen

- Divide

- Citizenship

- Communication

- Consumption

- Digital

- Education

- ICT

- Inclusion

- Information

- Interaction

- Interactivity

- Internet

- Journalism

- Knowledge

- Learning

- Literacy

- Mass

- Media

- Network

- Online Web

- Participation

- Press

- Skills

- Smartphone

- Usability

Finalmente, a través de una lectura de los títulos, palabras clave y abstracts, se verifican los artículos que aparecen en la lista de resultados y se archivan en Mendeley aquellos que realmente respondan a los criterios de interés de la presente investigación. Después de aplicar los criterios de selección y filtros mencionados, los resultados se reducen a 167 , que representa el 11,4\% de los 1.460 artículos publicados en el período de observación. 
Los artículos recopilados son procesados a través de Excel, para identificar características como: número de autores, institución de afiliación, país de procedencia, idioma, antigüedad y periodicidad. Se elaboran tablas y gráficos para visualizar los datos. Se utiliza el programa NVIVO11, para el trabajo de análisis de los textos de las palabras clave, los resúmenes y los abstracts (para los cuarenta artículos editados en Brasil que no presentan resumen en español). Se establece como criterio el análisis de las cien palabras más frecuentes en cada uno de los tres textos, se depura el listado eliminando palabras vacías, como adjetivos, conectores y artículos. Se elaboran nubes de palabras para el análisis cualitativo de contenido de los textos, con el propósito de identificar tópicos y enfoques predominantes. Finalmente, a través de una lectura de los resúmenes de los 167 artículos, se identifican los enfoques temáticos, el tipo de investigación, las técnicas utilizadas y las fuentes consultadas.

\section{Selección de la muestra de revistas y artículos}

A través de la consulta de Scimago ${ }^{(g)}$, se encuentran 22.856 revistas en Scopus, de las cuales el 16,4\% son de libre acceso. Incluye en el área de Ciencias Sociales: 4.828 revistas, de las cuales 173 corresponden a Latinoamérica. En la categoría "Comunicación”, se encuentran 256 revistas, que se distribuyen geográficamente de la siguiente manera:
$\diamond \quad 40 \%$ son del Reino Unido
$\diamond \quad 29 \%$ de Estados Unidos
$\checkmark \quad 7 \%$ de Holanda
$\diamond \quad 4 \%$ de Alemania
$\diamond \quad 4 \%$ de España
$\diamond \quad 2 \%$ de Brasil
$\diamond \quad 2 \%$ de Francia (hasta acá se acumula el $88 \%$ de las revistas).

Dada su distribución, predomina el idioma inglés. De las 256 revistas de Comunicación, 28 son de libre acceso (casi el 11\%); mientras que las demás revistas corresponden a editoriales comerciales ${ }^{13}$.

Las nueve revistas latinoamericanas especializadas en comunicación, incluidas en Scopus, son editadas por universidades y están a disposición de los usuarios en la web en acceso abierto. Esta es una tendencia importante en la región, cuyas revistas representan el 32\% de las revistas de Comunicación de acceso abierto en Scopus. Se destaca que nueve revistas españolas de Comunicación se encuentran en Scopus, de las cuales tres son de libre acceso.

Por otra parte, se hizo una búsqueda en la versión 2016 del Journal Citation Reports, en SSCI (Social Science Citation Report), en el Web of Science ${ }^{(\mathrm{h})}$, en las revistas clasificadas en la categoría "Comunicación”. Se encuentran 79 revistas. Solamente se incluye una española, la Revista Comunicar, editada en España por el Grupo Comunicar. De estas revistas, 76 se editan en inglés, una en holandés y dos son multilingües. Solamente seis revistas son de acceso abierto. No se encuentran revistas latinoamericanas de comunicación en la base de datos ISI Web of Science, sin embargo, (g) Scimago Journal \& Country Rank - https://www.scimagojr. com/journalrank.php

\footnotetext{
(h) Web of Science. Journal citation reports - http://jcr. incites.thomsonreuters.com/ JCRCategoryProfileAction. action?year=2016\&categoryName=COMMUNICATION\&edition=SSCI\&category=EU
} 
esta plataforma incluye recientemente el recurso Scielo Citation Index, para ampliar su cobertura a América Latina.

Dos de las revistas latinoamericanas que se encuentran en Scopus, están incluidas en Web of Science, pero no en la categoría "Comunicación", sino en "Information science and library”, en Q4: Transinformação y Informação e Sociedade. De esta manera, se plantea la complejidad de encontrar todas las publicaciones científicas de la región que divulguen investigaciones en el campo de la comunicación, al existir diversidad de categorías para la clasificación de estas, dada la necesidad de interdisciplinariedad propia del estudio de problemáticas sociales.

\section{Las revistas latinoamericanas de comunicación}

Las revistas especializadas seleccionadas en el presente estudio son las que están incluidas en Scopus, en la categoría “comunicación”. El Scimago Journal Rank (SJR) clasifica las revistas, de cada área, por cuartiles, del Q4 al Q1, siendo Q1 el de un impacto superior, de manera que todas las revistas del área se ordenan en cuatro partes o cuartiles. Por país de origen, se encuentran cinco revistas de Brasil, dos de Colombia, uno de México y uno de Chile. Todas son editadas por instituciones universitarias: cinco públicas y cuatro privadas. Las nueve revistas seleccionadas se encuentran en acceso abierto, a través de Internet y diferentes repositorios digitales, los cuales permiten aumentar la visibilidad y el acceso a estas publicaciones, a través de motores de búsqueda, como es el caso de Scielo ${ }^{(i)}$ y Redalyc ${ }^{(j)}$. En el caso de Scielo, siete de estas revistas se encuentran también en este repositorio (excepto Informação e Sociedade y Discursos Fotográficos). Mientras que para el caso de Redalyc se encuentran seis de estas revistas (no incluye: Informação e Sociedade, Discursos Fotográficos, ni Perspectivas em Ciencia da Informação).

Otro indicador que es importante para analizar la visibilidad y el impacto de las publicaciones científicas es el índice H-5, elaborado por Google $S c h o l a r^{(k)}$, indicador que sistematiza las citaciones de los artículos de las revistas durante los últimos cinco años, en Google Scholar, de la siguiente manera: Es el mayor número h de artículos publicados en los últimos cinco años que han recibido al menos h número de citaciones cada uno, de manera que un índice veinte quiere decir que durante los últimos cinco años la revista tiene veinte artículos que han sido citados por lo menos veinte veces cada uno ${ }^{(1)}$.

En relación con el índice H-5 de las revistas seleccionadas, merece la pena destacar que estas revistas han venido ganando visibilidad e impacto. La que tiene un índice mayor es Interface, con veinte y en el otro extremo se ubica Discursos Fotográficos, con cinco. Lo cual es consistente con su cuartil en la base de datos de Scopus. Las pocas citas que algunas de las revistas han obtenido podrían apuntar hacia la inestabilidad en su indización. Para tener un punto de referencia, la revista Comunicar, que está en Q1, tiene un índice H-5 de 33. Delgado y Repiso ${ }^{14}$ afirman que el índice h es una herramienta fiable y válida para medir el impacto de una revista. En la tabla 1, se observa una síntesis de la información de las revistas seleccionadas en el estudio. (i) Scientific Electronic Library Online. Periódicos por asunto, Ciências Sociais Aplicadas https://goo.gl/5bqCfX

(i) Sistema de Información Científica Redalyc - http://www. redalyc.org.

(k) Google Scholar Metrics https://goo.gl/WRpzmd.

(I) American University Library. Scholarly Research Impact Metrics - https://subjectguides library.american.edu/c php?g=175335\&p=1154177 
Tabla 1. Revistas seleccionadas

\begin{tabular}{|c|c|c|c|c|c|c|c|}
\hline Revista & Institución & País & Antigüedad & H5-Index & Q Scopus & Scielo & Redalyc \\
\hline Palabra Clave & $\begin{array}{c}\text { Universidad de La } \\
\text { Sabana }\end{array}$ & Colombia & 21 años & 11 & Q2 & $x$ & $x$ \\
\hline $\begin{array}{l}\text { Interface: Comunicação, } \\
\text { Saude, Educação }\end{array}$ & $\begin{array}{c}\text { Universidad Estatal } \\
\text { Paulista }\end{array}$ & Brasil & 20 años & 20 & Q2 & $x$ & $x$ \\
\hline Transinformação & $\begin{array}{l}\text { Pontificia Universidade } \\
\text { Catolica de Campinas }\end{array}$ & Brasil & 28 años & 9 & Q3 & $x$ & $x$ \\
\hline Cuadernos.info & $\begin{array}{l}\text { Pontificia Universidad } \\
\text { Católica de Chile }\end{array}$ & Chile & 33 años & 10 & Q3 & $x$ & $x$ \\
\hline $\begin{array}{c}\text { Perspectivas em } \\
\text { Ciencia da Informação }\end{array}$ & $\begin{array}{l}\text { Universidade Federal } \\
\text { de Minas Gerais }\end{array}$ & Brasil & 45 años & 9 & Q3 & $x$ & \\
\hline $\begin{array}{l}\text { Comunicación y } \\
\text { Sociedad (México) }\end{array}$ & $\begin{array}{l}\text { Universidad de } \\
\text { Guadalajara }\end{array}$ & México & 30 años & 8 & Q3 & $x$ & $x$ \\
\hline Informação e Sociedade & $\begin{array}{l}\text { Universidade Federal } \\
\text { de Campina Grande }\end{array}$ & Brasil & 26 años & 7 & Q3 & & \\
\hline Signo y Pensamiento & $\begin{array}{c}\text { Pontificia Universidad } \\
\text { Javeriana }\end{array}$ & Colombia & 36 años & 9 & Q4 & $x$ & $x$ \\
\hline Discursos Fotográficos & $\begin{array}{l}\text { Universidade Estadual } \\
\text { de Londrina }\end{array}$ & Brasil & 12 años & 5 & Q4 & & \\
\hline
\end{tabular}

Fuente: Elaboración propia, a partir de la consulta de los recursos citados.

Otra característica de estas revistas que merece la pena destacar es su antigüedad, dado que ocho revistas tienen más de veinte años de labores y "Discursos Fotográficos" se publica hace 12 años.

\section{Artículos de investigación seleccionados para su análisis}

Se recopilaron 167 artículos de investigación de las nueve revistas de comunicación latinoamericanas mencionadas anteriormente. Estos artículos son editados en Brasil (34\%), Colombia (29\%), Chile (20\%) y México (17\%). Brasil ocupa el primer lugar, dado que cinco de las nueve revistas son brasileras. En la tabla dos pueden observarse la relación de artículos seleccionados, publicados durante los cinco últimos años.

Tabla 2. Artículos de investigación seleccionados

\begin{tabular}{|c|c|c|c|c|c|}
\hline Revista & Perfil editorial declarado & Periodicidad & $\begin{array}{l}\text { Total artículos } \\
\text { 2013-2017 }\end{array}$ & $\begin{array}{l}\text { Artículos } \\
\text { seleccionados }\end{array}$ & $\%$ \\
\hline Palabra Clave & $\begin{array}{l}\text { Investigación y reflexión en los distintos } \\
\text { campos de la comunicación }\end{array}$ & Trimestral & 168 & 29 & 20,8 \\
\hline $\begin{array}{l}\text { Interface: Comunicação, } \\
\text { Saude, Educação }\end{array}$ & $\begin{array}{l}\text { Interdisciplinar, priorizando la relación de la } \\
\text { comunicación y/o educación con la salud }\end{array}$ & Trimestral & 458 & 11 & 2,4 \\
\hline Transinformação & $\begin{array}{l}\text { Ciencia de la Información y } \\
\text { Biblioteconomía y áreas afines. }\end{array}$ & Cuatrimestral & 88 & 14 & 15,9 \\
\hline Cuadernos.info & $\begin{array}{l}\text { Investigaciones sobre comunicación } \\
\text { mediada en Iberoamérica }\end{array}$ & Semestral & 107 & 34 & 31,8 \\
\hline $\begin{array}{c}\text { Perspectivas em } \\
\text { Ciencia da Informação }\end{array}$ & $\begin{array}{l}\text { Ciencia de la Información y } \\
\text { Biblioteconomía }\end{array}$ & Trimestral & 232 & 5 & 2,2 \\
\hline $\begin{array}{l}\text { Comunicación y } \\
\text { Sociedad (México) }\end{array}$ & $\begin{array}{l}\text { Investigaciones en el campo de la } \\
\text { comunicación social }\end{array}$ & Cuatrimestral & 93 & 28 & 30,1 \\
\hline
\end{tabular}


Tabla 2. continuación

\begin{tabular}{|c|c|c|c|c|c|}
\hline Revista & Perfil editorial declarado & Periodicidad & $\begin{array}{l}\text { Total artículos } \\
\text { 2013-2017 }\end{array}$ & $\begin{array}{l}\text { Artículos } \\
\text { seleccionados }\end{array}$ & $\%$ \\
\hline $\begin{array}{l}\text { Informacao e } \\
\text { Sociedade: Estudos }\end{array}$ & $\begin{array}{l}\text { Ciencia de la Información y } \\
\text { Biblioteconomía y áreas afines. }\end{array}$ & Cuatrimestral & 154 & 22 & 14,3 \\
\hline Signo y Pensamiento & $\begin{array}{l}\text { Investigaciones en los campos de la } \\
\text { comunicación, la información y el lenguaje }\end{array}$ & Semestral & 57 & 19 & 33,3 \\
\hline \multirow[t]{2}{*}{ Discursos Fotográficos } & $\begin{array}{l}\text { Investigaciones en el campo de la } \\
\text { comunicación visual }\end{array}$ & Semestral & 103 & 5 & 4,9 \\
\hline & Total & & 1460 & 167 & 11,4 \\
\hline
\end{tabular}

Fuente: Elaboración propia

Como se observa en la tabla anterior, las revistas Transinformação y Informação e Sociedade, que en Web of Science están clasificadas en la categoría: "Information science and library", acogen artículos de investigación en el campo de la comunicación, educomunicación y periodismo, en la primera éstos representan el 15,9\% y en la segunda son el $14 \%$. Se destaca que todas las revistas publican varios números al año y un volumen considerable de artículos de investigación. En el caso de la revista Interface $^{(\mathrm{m})}$, ésta presenta el mayor volumen de artículos publicados durante el período de observación.

\section{Resultados}

En cuanto a la procedencia de los artículos seleccionados, se destacan las contribuciones desde Brasil, con un 31,1\%, seguido de España, con el 25,8\% y México y Colombia, cada uno con el 12\%, Chile con el 7,2\% y Argentina con el 6,6\%.

En cuanto a la publicación de artículos provenientes del mismo país de origen de la editorial de la revista, se destaca en el caso de Brasil, que el 82,5\% de los artículos provienen del mismo país. Esto también se explica por la existencia de menos revistas en portugués. En México, el $43 \%$ de los artículos publicados provienen del mismo país. En Colombia, el 35\% de los artículos publicados provienen del mismo país y en Chile, las contribuciones del mismo país representan el $21 \%$ de los artículos publicados.

En cuanto a idiomas, predomina la lengua materna propia del país en el cual es editada la revista, el portugués, en el caso de Brasil ( $83 \%$ de los artículos) y el español para Colombia, Chile y México (93\% de los artículos). Lo que también coincide con el hecho de que España sea el segundo país de origen de las contribuciones publicadas en las revistas seleccionadas. Los artículos en inglés representan el 5\%.

En relación con la autoría de los artículos seleccionados, se destaca que el $41 \%$ de los artículos son elaborados por un autor, el 30\% por dos autores y el 19\% por tres autores. En total, se cuentan 341 autores involucrados en los 167 artículos de la muestra.

En años recientes, se identifica la tendencia de aumento en el número de autores de los artículos publicados, ya que en 2016 y 2017 disminuyen los artículos elaborados por un solo autor al 34,4\% y aumentan los artículos elaborados por tres autores hasta representar el 26,6\% de los artículos recopilados.

En cuanto a las instituciones de afiliación, predominan los artículos provenientes de una única institución, con el 66\%, proporción mayor a la de los artículos elaborados por un único autor, lo que evidencia la existencia de artículos producidos por varios (m) Revista Interface: Comunicação, Saúde, Educação - http:// interface.org.br/es/a-revista/ 
autores de una misma entidad, mientras el $23 \%$ son elaborados por autores de dos instituciones. En el 10,8\% de los artículos seleccionados, la segunda institución de afiliación es de otro país, predominando España con el 33\%, seguido de Colombia, con el 16,7\%, Chile con el $11 \%$ y EE. UU. con el $11 \%$.

\section{Análisis de contenido de los artículos}

A través del software NVIVO 11, se elaboran nubes de palabras de los contenidos de los artículos: las palabras clave, los resúmenes y abstracts. Las nubes de palabras son una representación visual de las palabras utilizadas con mayor frecuencia en el texto, de manera que el gráfico muestra las palabras más relevantes con un tamaño mayor. Son un recurso para el análisis cualitativo de contenido, al destacar los tópicos relacionados con el tema de interés.

En la Figura 1, se visualizan las palabras claves designadas por los autores de los artículos seleccionados.

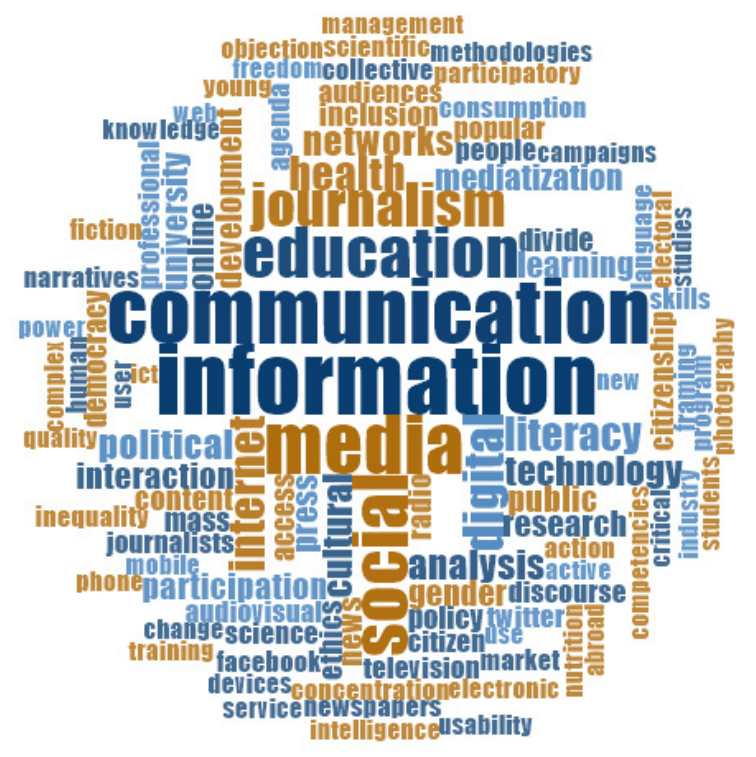

Figura 1. Nube de palabras de frecuencia de keywords

Como se aprecia en la figura anterior, las palabras más utilizadas por los autores como keywords son: información, comunicación, social, medios (media), educación, periodismo, Internet, alfabetización (literacy), digital, salud y aprendizaje. Lo que evidencia el predominio de un enfoque temático en el cual se destacan los medios y los entornos digitales. Otros términos importantes se relacionan con el carácter investigativo de los artículos seleccionados, en los cuales se destacan: análisis, ciencia, científico, contenidos, públicos, audiencias, conocimiento, inteligencia, lenguaje, discurso, encuadre (framing) y universidad. En relación con los escenarios digitales, que son una tendencia importante en los contenidos, se destacan conceptos como: digital, en línea (online), red, brechas, interactividad, interacción, acceso, web, 
Facebook, Twitter y usabilidad. En el campo educativo, se destacan, además de las palabras mencionadas antes: participación, desarrollo, agenda, entrenamiento, mediación, servicio, público, colectivo, crítico, habilidades, programa, inequidad, ética, campaña y consumo.

A continuación, se analizan los contenidos de los 127 resúmenes que se encuentran en español, que se observan en la Figura 2:

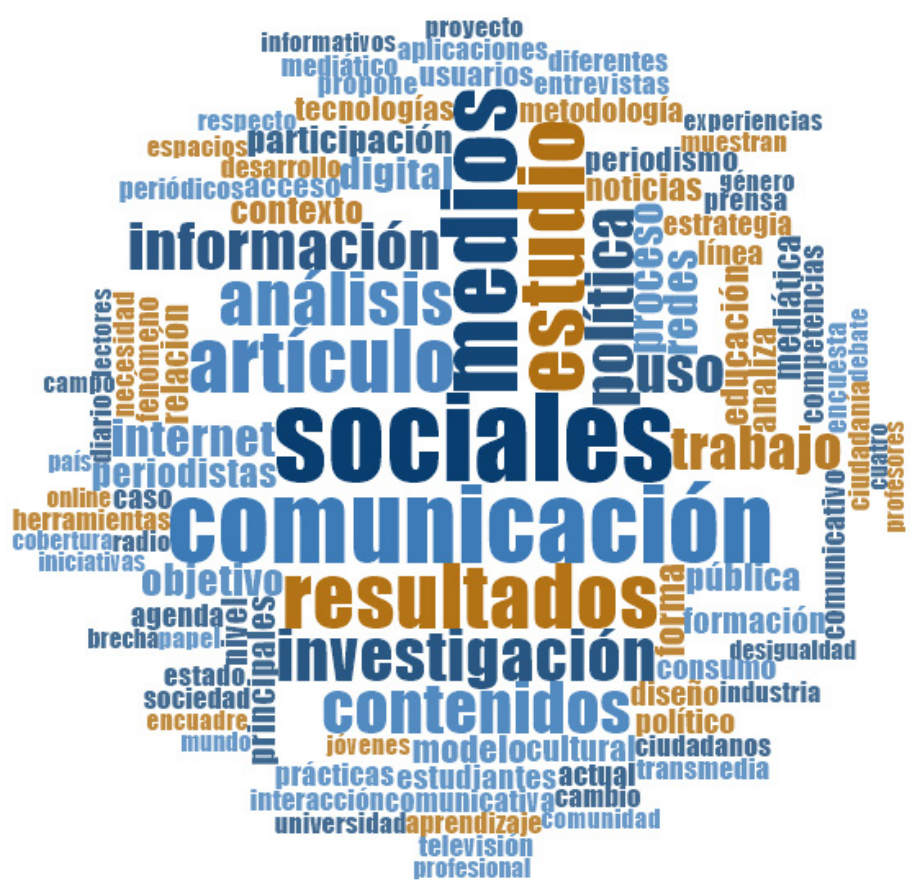

Figura 2. Nube de palabras de frecuencias resúmenes en español

Luego de eliminar las palabras vacías, las cien palabras con mayor frecuencia en los resúmenes de los artículos seleccionados son: comunicación, medios, sociales, análisis, información, investigación, trabajo, Internet, contenidos y social. En los resúmenes también puede identificarse que los entornos digitales son un tema central de los artículos seleccionados, del cual se destacan tópicos como: digital, uso, redes, usuarios, acceso, aplicaciones, transmedia, TIC, interacción, tecnologías, en línea. Relacionado con el tema educativo, se destacan las palabras: educación, aprendizaje, formación, cultural, relación, estudiantes, participación, competencias, alfabetización, universidad. En el gráfico, se observan enfoques del campo de la educación en el cual predomina lo tecnológico, lo digital y lo periodístico, con un énfasis en los aspectos sociales y en los actores involucrados.

A continuación, en la Figura 3 se presenta la visualización de los términos más frecuentes en los abstracts de cuarenta artículos editados en Brasil.

Como se puede ver en el gráfico, de los cuarenta resúmenes en inglés, se destacan los términos: información, investigación, social, salud, data, estudio, investigación, educación, uso, alfabetización, conocimiento, participación, proyecto y digital. A partir del gráfico, es posible identificar una tendencia importante en los artículos editados en Brasil hacia el campo de la salud y de la educación, desde una perspectiva 
popular, que predomina en los abordajes de la relación comunicación-educación desde Latinoamérica ${ }^{15}$.

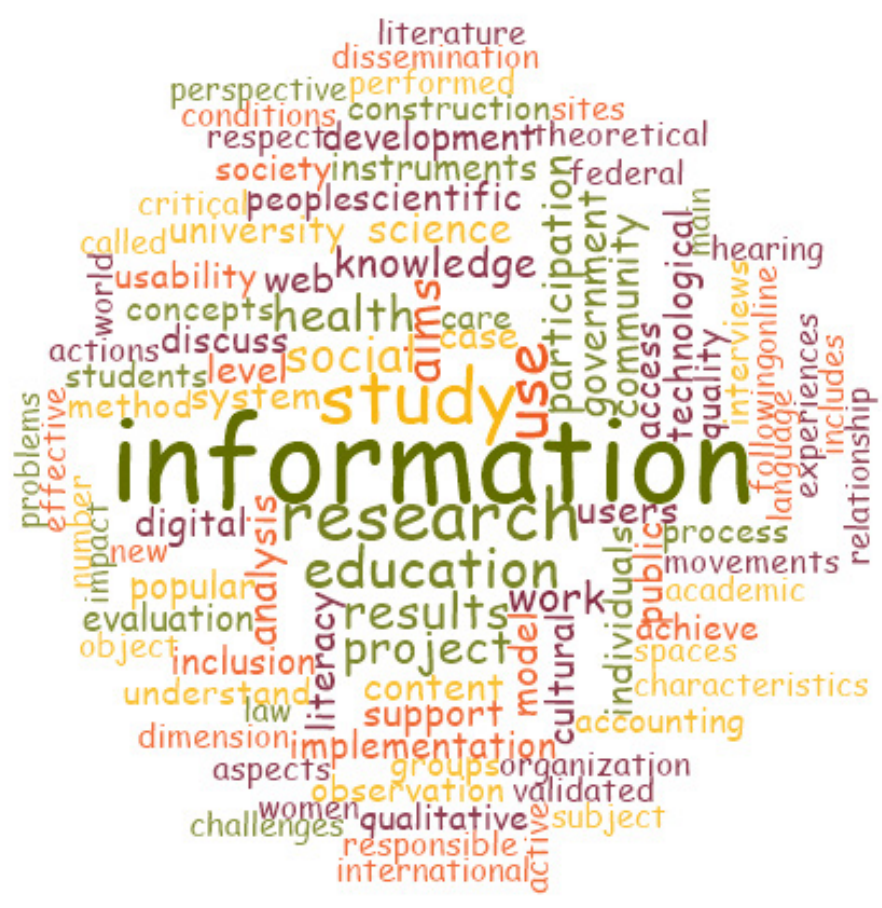

Figura 3. Nube de palabras de abstracts

A partir de una lectura de los abstracts de los 167 artículos recopilados, se identifican otras características importantes, como los enfoques que predominan en el abordaje de la temática de cada artículo, el método de investigación y las técnicas de investigación aplicadas, fuente de información o población consultada. En algunas ocasiones, cuando el resumen no responde por la información de interés, se revisan los artículos completos. Luego de la lectura, se clasifican los contenidos por categorías. En el Grafico 1, puede observarse los enfoques predominantes.

Se destaca que el 35\% de los artículos encuadran sus objetos de estudio desde la comunicación digital, siendo el enfoque que más se encuentra en los artículos de las revistas latinoamericanas analizadas, seguido por objetos con mayor tradición en el campo de la comunicación en Latinoamérica, como el periodismo y medios masivos. Merece la pena considerar que el tema de brecha digital ocupa el cuarto lugar, con el 6\%, que se pueden sumar al marco de la comunicación digital como enfoque predominante en los artículos publicados durante los últimos cinco años.

En relación con el abordaje de la relación comunicación-educación, se identifica como enfoque predominante en el $15 \%$ de los artículos recopilados, incluyendo perspectivas como la educación para la salud, la educación, educomunicación y alfabetización mediática. En los artículos recopilados, los autores diferencian entre competencia mediática y competencia informacional, si bien los componentes informacionales así como los digitales forman parte de una definición más integral de la competencia mediática ${ }^{16}$. 


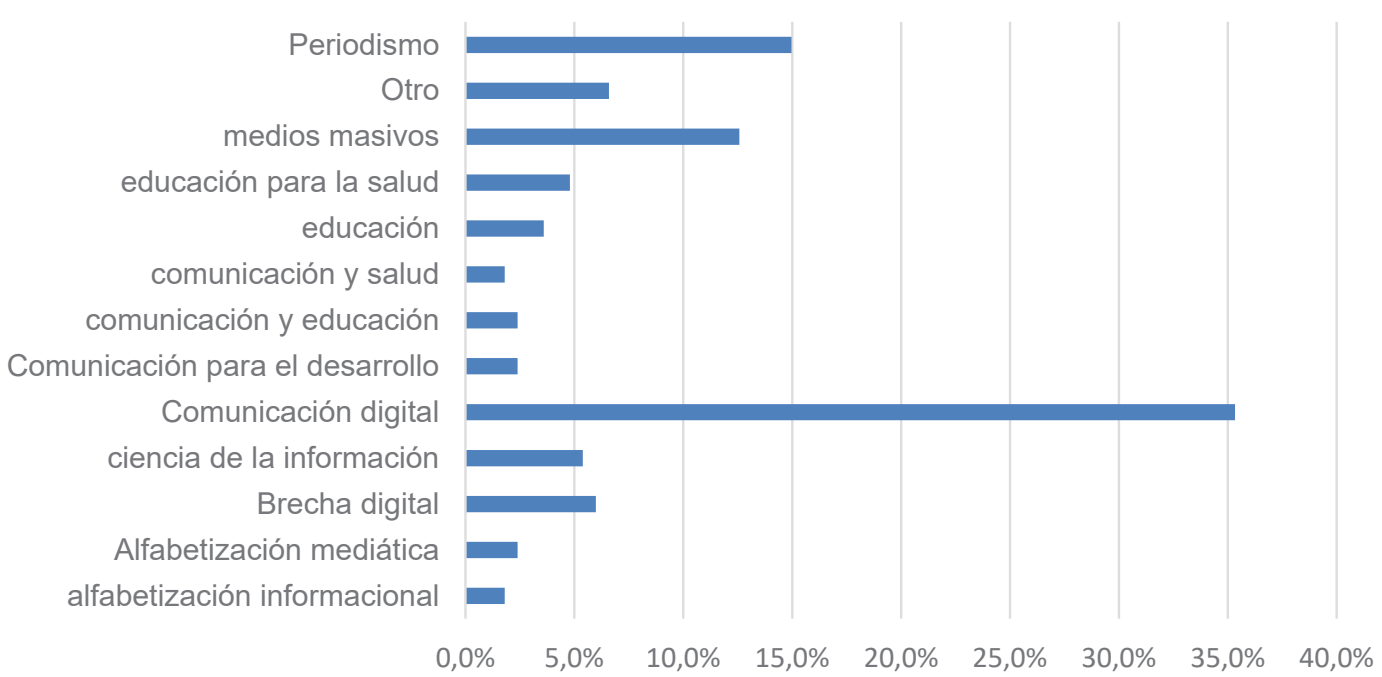

Gráfico 1. Enfoque predominante en el abordaje del tema

Fuente: Elaboración propia

En cuanto al tipo de investigación que se presenta en los artículos, predomina la investigación cualitativa, propia de las ciencias sociales y de la comunicación, en el 41,9\% de los artículos, seguida por la investigación cuantitativa, con el $25,7 \%$, la teórica con el 14,4\% y autodenominados ensayos con el 6,6\%. Empieza a destacarse la presencia de estudios de diseño mixto, con el 11,4\%, los cuales reconocen la naturaleza complementaria de los métodos de investigación, ante la complejidad de las problemáticas de estudio.

En las investigaciones cualitativas predominan como técnicas para la recopilación de la información: la entrevista, la observación y el estudio de casos, en su orden. Mientras que para los estudios cuantitativos, las principales técnicas de investigación son: la encuesta y el análisis de contenido.

En los artículos recopilados predominan los usuarios o audiencias, como fuente de información o población consultada, en el 20,4\%. Se destacan las fuentes secundarias con el 18,6\%, en el caso de los artículos resultado de investigaciones teóricas y en los ensayos. En el caso de los artículos resultado de investigaciones aplicadas, las principales fuentes consultadas son los medios (15,6\%), los cibermedios $(6,6 \%)$, los contenidos $(11,4 \%)$ y los periodistas $(7,8 \%)$. Mientras que, como públicos involucrados en procesos educativos, los más consultados son los estudiantes $(10,8 \%)$ y educadores $(4,2 \%)$.

\section{Conclusiones}

A partir del análisis de los artículos recopilados, se confirma que la comunicación digital, incluyendo la brecha digital, es el encuadre temático central, por encima de otras perspectivas sociales, culturales, de los medios y de los estudios del desarrollo, con gran tradición en la investigación latinoamericana de la comunicación. Los escenarios digitales confirman el interés que se identifica en los artículos de la muestra en procesos de alfabetización mediática, la cual actualiza la discusión sobre competencias informacionales, comunicativas, audiovisuales y digitales, fundamentales en los 
procesos educomunicativos que tienen la finalidad de mejorar las capacidades de los ciudadanos como usuarios, consumidores y productores de contenidos de distinta índole. Este proceso remite a una realidad compleja y multidimensional, que incorpora a diversos actores en su campo de interés, los cuales interactúan de manera dinámica en la construcción y fortalecimiento de las capacidades, habilidades y desempeños que exige el mundo digital a los ciudadanos de la aldea global, para estar habilitados como ciudadanos, consumidores, productores y usuarios de los contenidos, dispositivos y herramientas, en entornos de hiperabundancia informativa y para asumir un rol más activo como ciudadanos del mundo.

A pesar de predominar el interés por el campo de la comunicación digital, se encuentra un abordaje menor de objetos de estudio novedosos, como las narrativas transmedia y los MOOC (Massive Online Open Courses), los cuales tienen un gran desarrollo actualmente, ya que permiten responder a la demanda social de una educación a lo largo de la vida, potenciada por los escenarios digitales ${ }^{17}$.

En el proceso de análisis de contenido de los artículos de la muestra, también se identifican enfoques propios de la relación comunicación-educación, desde el contexto latinoamericano de la comunicación-educación, entre los cuales sobresale el enfoque en procesos de educomunicación para la salud.

En el estudio se destaca que el $41 \%$ de los artículos son elaborados por un autor; ante esta gran proporción, Barranquero y Ángel afirman ${ }^{18}$ que permite identificar "la difícil situación que aún se vive en muchas universidades latinoamericanas en términos de su estructura de investigación” (p. 45).

En la región iberoamericana, la publicación en revistas de alto impacto es un criterio fundamental en la evaluación de la calidad de la educación en las universidades y cada vez más importante en la acreditación, evaluación y ascenso de profesores e investigadores, por parte de las agencias oficiales, que son cada vez más exigentes. Mancinas-Chávez, Romero-Rodríguez y Aguaded ${ }^{19}$ llaman la atención en cuanto a la existencia de pocos espacios para la publicación de investigaciones en comunicación en español en revistas de alto impacto, dado que como se ha mencionado antes, en Scopus se incluyen 13 revistas en español (nueve revistas españolas, dos de Colombia, una de Chile, una de México), las cuales se encuentran sobresaturadas de solicitudes, porque reciben artículos de investigadores de los 18 países latinoamericanos hispanohablantes y de España, lo cual incide en la tasa de rechazo de artículos.

Mientras que en la muestra analizada solamente se encuentra un 5\% de artículos en inglés, ante la escasez de espacios para la visibilización de los resultados de las investigaciones realizadas en las universidades iberoamericanas, Mancinas-Chávez, Romero-Rodríguez y Aguaded destacan que, dado que los espacios de divulgación científica son insuficientes, la comunidad académica se ve forzada a publicar en revistas internacionales de habla inglesa, las cuales también pueden incidir en la construcción de una agenda de temas, así como también en el predominio de abordajes metodológicos cuantitativos, que representan un porcentaje considerable de los artículos analizados y que, sin duda, cobrará una importancia creciente en el campo de las publicaciones científicas. 


\section{Contribuciones de los autores}

Todos los autores participaron activamente de todas las etapas de elaboración del manuscrito.

\section{Agradecimientos}

Este trabajo se ha elaborado en el marco de Alfamed (Red Interuniversitaria Euroamericana de Investigación en Competencias Mediáticas para la Ciudadanía), con el apoyo del Proyecto I+D+I Coordinado "Competencias mediáticas de la ciudadanía en medios digitales emergentes (smartphones y tablets): practicas innovadoras y estrategias educomunicativas en contextos múltiples” (EDU2015-64015-C3-1-R) (MINECO/ FEDER), y de la "Red de Educación Mediática” del Programa Estatal de Investigación Científica-Técnica de Excelencia, Subprograma Estatal de Generación de Conocimiento (EDU2016-81772-REDT), financiados por el Fondo Europeo de Desarrollo Regional (FEDER) y Ministerio de Economía y Competitividad de España.

\section{Derechos de autor}

Este artículo está bajo la Licencia Internacional Creative Commons 4.0, tipo BY (https:// creativecommons.org/licenses/by/4.0/deed.es).

(cc)

\section{Referencias}

1. Pereira J. La comunicación: un campo de conocimiento en construcción. Reflexiones sobre la comunicación social en Colombia. Investig Desarro. 2005; 13(2):412-42.

2. González-Samé H, Romero-Rodríguez LM, Aguaded I. La investigación en comunicación en Latinoamérica: una aproximación histórica. Hist Comun Soc. 2017; 22(2):427-43.

3. Martín-Barbero J. De los medios a las mediaciones comunicación, cultura y hegemonía. Bogotá: Anthropos; 2010.

4. Martín-Barbero J. ¿Desde dónde pensamos la comunicación hoy? Chasqui Rev Latinoam Comun. 2015; (128):13-29.

5. Beltrán L. La comunicación para el desarrollo en Latinoamérica: un recuento de medio siglo. Anagramas. 2015; 4(8):53-76.

6. Scolari C. Hipermediaciones elementos para una teoría de la comunicación digital interactiva. Barcelona: Gedisa; 2008.

7. Fundación Telefónica. La Sociedad de la Información en España 2016. Madrid: Ariel; 2017.

8. Romero-Rodríguez LM, Aguaded I. Consumo informativo y competencias digitales de estudiantes de periodismo de Colombia, Perú y Venezuela. Convergencia. 2016; $23(70): 35-57$.

9. United Nations Educational, Scientific and Cultural Organization. La educación en materia de comunicación. París: Unesco; 1984. 
10. Oliveira-Soares I. El derecho a la pantalla: de la educación en medios a la educomunicación en Brasil. Comunicar. 2008; 31:77-82. doi: 10.3916/c31-2008-01009.

11. Marta-Lazo C. La educación en materia de comunicación, una asignatura pendiente. Ámbitos. 2008; (17):225-36.

12. Ferrés J, Piscitelli A. La competencia mediática: propuesta articulada de dimensiones e indicadores. Comunicar. 2012; 19(38):75-82. doi: 10.3916/C38-2012-02-08.

13. Rogel-Salazar R, Santiago-Bautista I, Martínez-Domínguez N. Revistas científicas latinoamericanas de Comunicación indizadas en WoS, Scopus y bases de datos de Acceso Abierto. Comun Soc. 2017; (30):167-96.

14. Delgado E, Repiso R. El impacto de las revistas de comunicación: comparando Google Scholar Metrics, Web of Science y Scopus. Comunicar. 2013; 21(41):45-52. doi: 10.3916/C41-2013-04.

15. Rivera-Rogel D, Zuluaga-Arias L, Ramírez N, Romero-Rodríguez L, Aguaded I. Media competencies for the citizenship training of teachers from Andean America: Colombia and Ecuador. Paidéia (Ribeirão Preto). 2017; 27(66):80-9. doi: $10.1590 / 1982-43272766201710$.

16. Pérez MA, Delgado A. De la competencia digital y audiovisual a la competencia mediática: dimensiones e indicadores. Comunicar. 2012; 20(39):25-34. doi: 10.3916/ C39-2012-02-02.

17. Osuna-Acedo S, Marta-Lazo C, Frau-Meigs D. De sMOOC a tMOOC, el aprendizaje hacia la transferencia profesional: el proyecto europeo ECO. Comunicar. 2018; 26(55):105-14. doi: 10.3916/C55-2018-10.

18. Barranquero A, Ángel A. La producción académica sobre comunicación, desarrollo y cambio social en las revistas científicas de América Latina. Signo Pensam. 2015; 67(34):30-58. doi: 10.11144/Javeriana.syp34-67.pacd

19. Mancinas-Chávez RM, Romero-Rodríguez LM, Aguaded I. Problemas de la divulgación de las investigaciones en comunicación en revistas de alto impacto en español. F@Ro. 2016; 1(23):241-58.

This study analyzed recent research trends in communication research published in nine leading communication journals in Latin America. A literature review of scientific articles published between 2013 and 2017 was conducted using the Scopus database. A database query was run, together with the systematization of data using bibliographic management software and qualitative content analysis of selective texts using the QDA software NVivo 11. A total of 167 articles addressing the themes educommunication, media literacy, and transmedia narrative were analyzed. The majority of articles were written by individual authors belonging to a single institution and country, with emphasis on Brazil and Spain. With respect to content, the predominant themes were media and digital environments and the majority of studies used a qualitative approach.

Keywords: Communication. Education. Educommunication. Media literacy. Scientific journals. 
Analisam-se as tendências recentes de pesquisa em comunicação publicadas nas nove revistas de comunicação mais significativas da América Latina. A pesquisa se centra na revisão dos artigos científicos publicados entre 2013 e 2017 em Scopus. A metodologia se baseia na consulta de bases de dados, sua sistematização por meio de gestores bibliográficos e a analise do conteúdo qualitativo dos textos selecionados, com o uso do software QDA NVIVO11. Estudam-se 167 artigos que abrangem temáticas de educomunicação, alfabetização midiática e narrativas transmídia. Pode-se identificar o predomínio da autoria individual, assim como a filiação a uma só instituição e a um só país, com destaque para Brasil e Espanha. A respeito do conteúdo, predomina o foco temático sobre mídia e os entornos digitais, assim como uma abordagem qualitativa das pesquisas.

Palavras-chave: Comunicação. Educação. Educomunicação. Alfabetização midiática. Revista científica.

Presentado el 07/04/18.

Aprobado el 08/10/18. 